\title{
Independent and reproducible hippocampal radiomic biomarkers for multisite Alzheimer's disease: diagnosis, longitudinal progress and biological basis
}

Kun Zhao

Shandong normal university

Yanhui Ding

Shandong normal university

Ying Han

Xuanwu Hospital

Yong Fan

University of Pennsylvania

Aaron F. Alexander-Bloch

Yale University School of Medicine

Tong Han

Tianjin Huanhu Hospital

Dan Jin

Institute of Automation Chinese Academy of Sciences

Bing Liu

Institute of Automation Chinese Academy of Sciences

Jie Lu

Xuanwu Hospital

Chengyuan Song

Shandong University Qilu Hospital

\section{Pan Wang}

Tianjin Huanhu Hospital

Dawei Wang

Shandong University Qilu Hospital

\section{Qing Wang}

Shandong University Qilu Hospital

\section{Kaibin Xu}

Institute of Automation Chinese Academy of Sciences

Hongwei Yang

Xuanwu Hospital 


\section{Hongxiang Yao}

Chinese PLA General Hospital

\section{Yuanjie Zheng}

Shandong normal university

\section{Chunshui Yu}

Tianjin Medical University General Hospital

\section{Bo Zhou}

Chinese PLA General Hospital

\section{Xinqing Zhang}

Xuanwu Hospital

\section{Yuying Zhou}

Tianjin Huanhu Hospital

\section{Tianzi Jiang}

Institute of Automation Chinese Academy of Sciences

\section{Xi Zhang}

Chinese PLA General Hospital

\section{Yong Liu ( $\nabla$ yliu@nlpr.ia.ac.cn )}

Institute of Automation Chinese Academy of Sciences https://orcid.org/0000-0002-1862-3121

\section{Research}

Keywords: Hippocampal radiomic features, Multisite Alzheimer's disease MRI, Independent crossvalidation, Brain biomarker, Biological basis

Posted Date: December 17th, 2019

DOI: https://doi.org/10.21203/rs.2.17749/v2

License: (c) (1) This work is licensed under a Creative Commons Attribution 4.0 International License. Read Full License

Version of Record: A version of this preprint was published at Science Bulletin on July 1st, 2020. See the published version at https://doi.org/10.1016/j.scib.2020.04.003. 
The authors have withdrawn this preprint from Research Square 


\section{Figures}

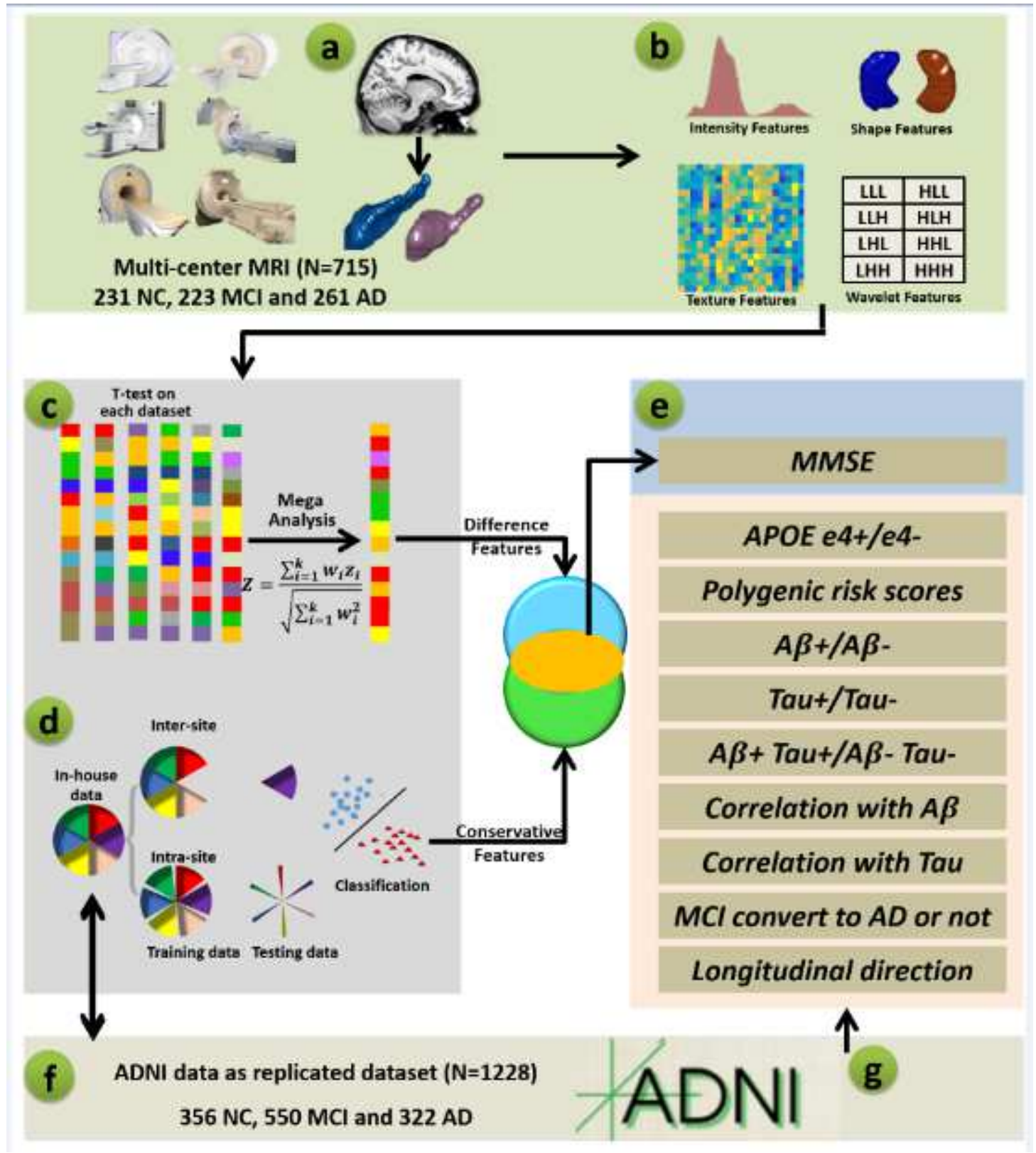

Figure 1

Schematic of the data analysis pipeline. (a) Automatic segmentation of the hippocampus in the individual space with the LLL method. (b) Computation of radiomic features. (c) T-test between NC and $A D$ and mega-analysis to identify differences at the multicenter level. (d) Classification analysis between $A D$ and $N C$ using inter-site and intra-site cross-validation to test the generalizability of the results. (e) Correlation between MMSE and radiomic features (and the decision value of the subject to the classification plane). (f) Validation of the ADNI dataset for using ADNI as training data with in-house data as testing data as well as using in-house as training data with ADNI data as testing data to test the generalizability of the results. (g) Relationship between radiomic features and clinical information (e.g., cognitive ability, APOE genetics, A, , Tau, and longitudinal changes in the cognitive abilities in $\mathrm{MCl}$ ). 


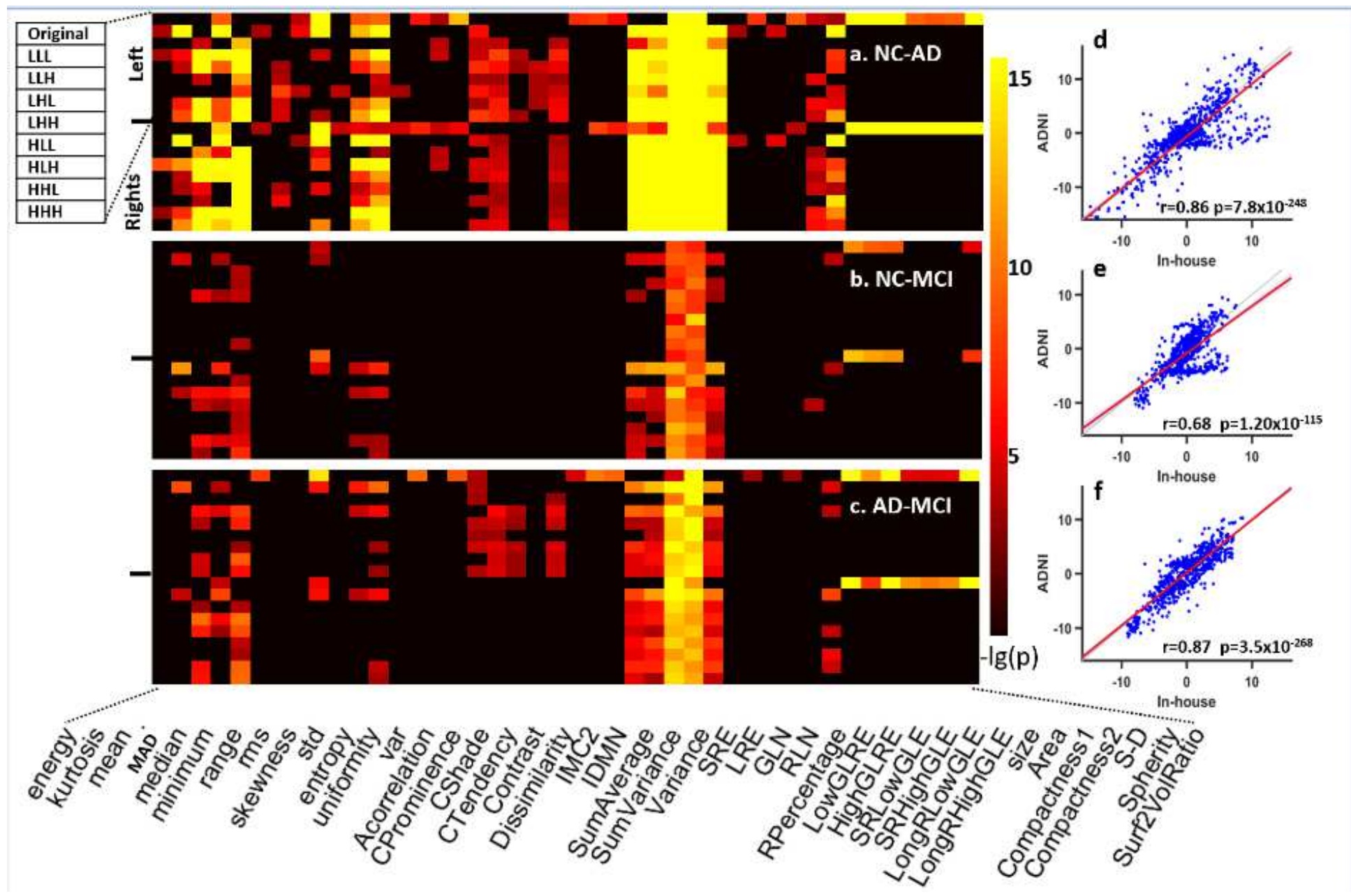

Figure 2

(a) Group differences of the used radiomic features between AD and NC. (b) Group differences of the used radiomic features between $\mathrm{NC}$ and $\mathrm{MCl}$. (c) Group differences of the used radiomic features between $A D$ and $\mathrm{MCl}$. For a-c, each matrix has 18 rows, of which the first 9 rows show the left hippocampus, and the next 9 rows show the right hippocampus; for each hemisphere, the first row represents the features in the raw space, and the following 8 rows represent the results for different frequency domains of the wavelet transform (LLL, LLH, LHL, HLL, HHL, LHH, HLH, HHH). The color bar represents $-\log (p)$, and $p$ is computed by a mega-analysis of the 6 sites. (d) Correlation between the inhouse statistical scores and the ADNI statistical scores of the used radiomic features in NC and AD. (e) Correlation between the in-house statistical scores and the ADNI statistical scores of the used radiomic features in $\mathrm{NC}$ and $\mathrm{MCl}$. (f) Correlation between the in-house statistical scores and the ADNI statistical scores of the used radiomic features in $A D$ and $\mathrm{MCl}$. 

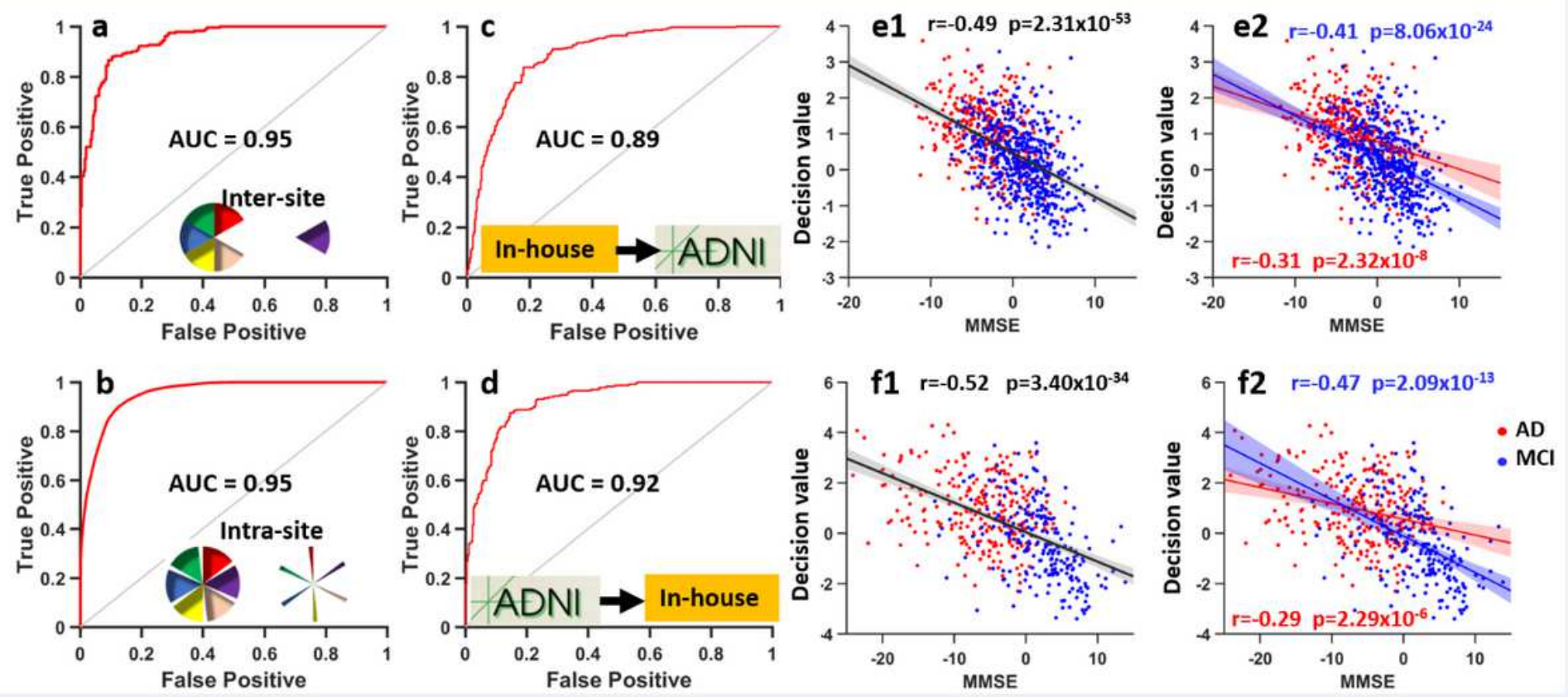

\section{Figure 3}

(a) The ROC curve for classification between AD and NC with inter-site cross-validation. (b) The ROC curve for classification between $A D$ and NC intra-site cross-validation. (c) The ROC curve for classification using in-house data as training data and ADNI data as test data. (d) The ROC curve for classification using ADNI data as training data and in-house data as testing data. (e) Correlation of classifier outputs with the MMSE score of the ADNI data (e1, the MCl group and AD group; $\mathrm{e} 2$, only in the AD group or only in the $\mathrm{MCl}$ group. (f) Correlation of classifier outputs with the MMSE score of the in-house data ( $\mathrm{f} 1, \mathrm{MCl}$ group and $A D$ group; $f 2$, only in the $A D$ group or only in the $\mathrm{MCl}$ group). 


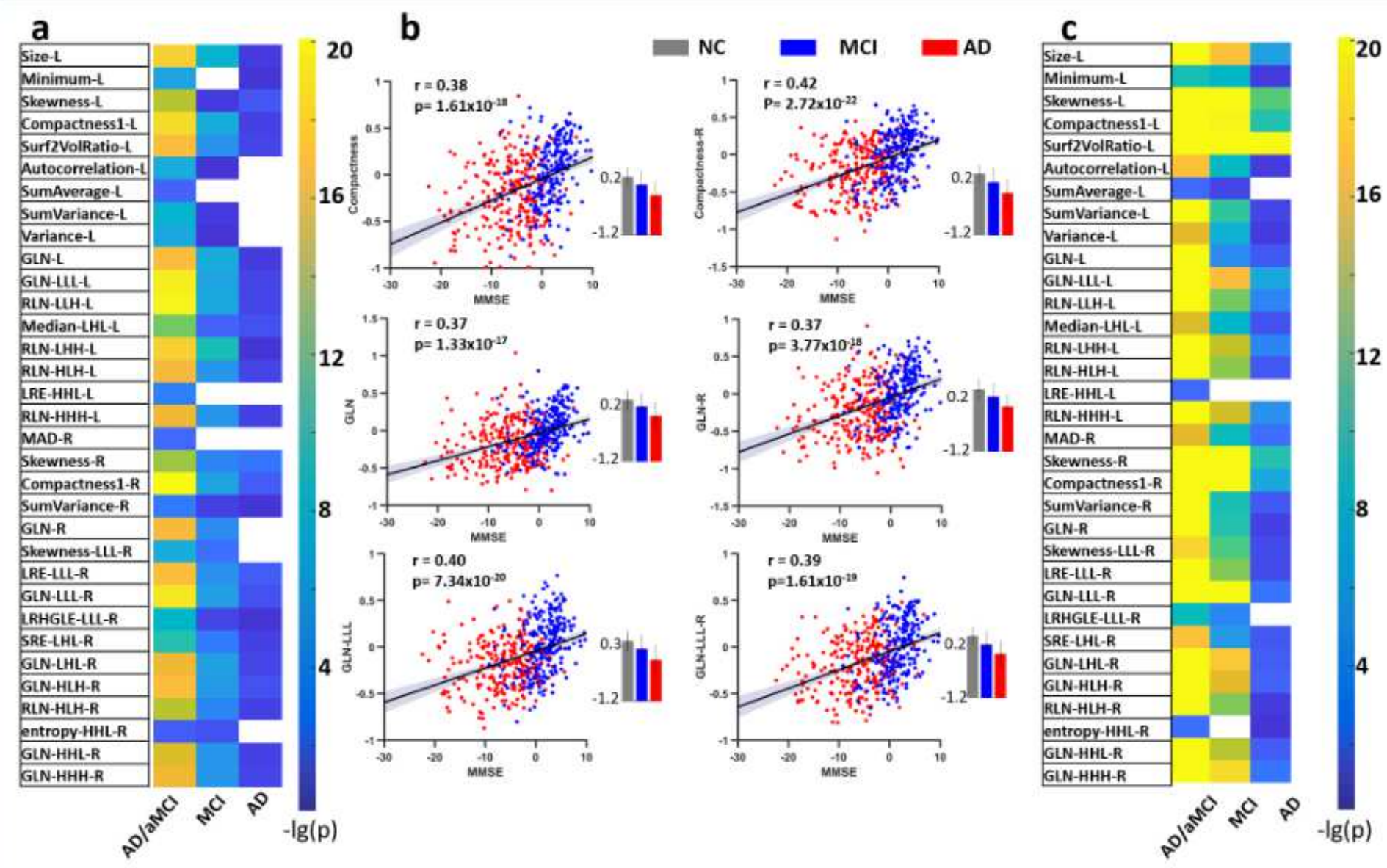

\section{Figure 4}

(a) Correlations between the MMSE score and a subset of radiomic features. The values of the color bar reflect $-\log (\mathrm{p})$, and the 3 columns are the combined $\mathrm{AD}$ and $\mathrm{MCl}$ group, only the $\mathrm{MCl}$ group and only the AD group. (b) Several significant correlations illustrated in scatter plots. (c) Correlation with the MMSE score and the radiomic features for the ADNI data. Abbreviations: $G L N=$ gray level nonuniformity, RLN=run length nonuniformity, LRE=long run emphasis, $\mathrm{SRE}=$ short run emphasis, Surf2VolRatio=ratio of surface area to volume, LRHGLE=long run high gray level emphasis. Additional information can be found in the supplementary materials. - $L$ means left hippocampus features, and $-R$ means right hippocampus features. 
a

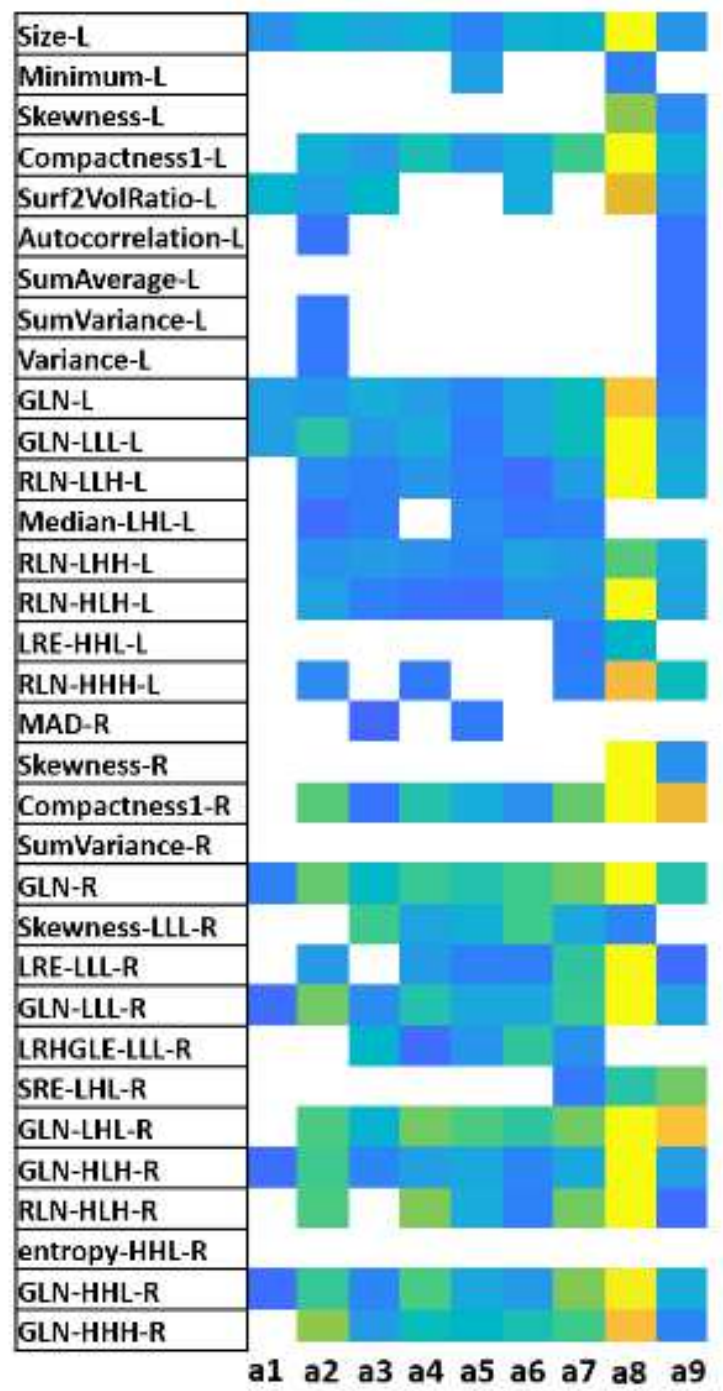

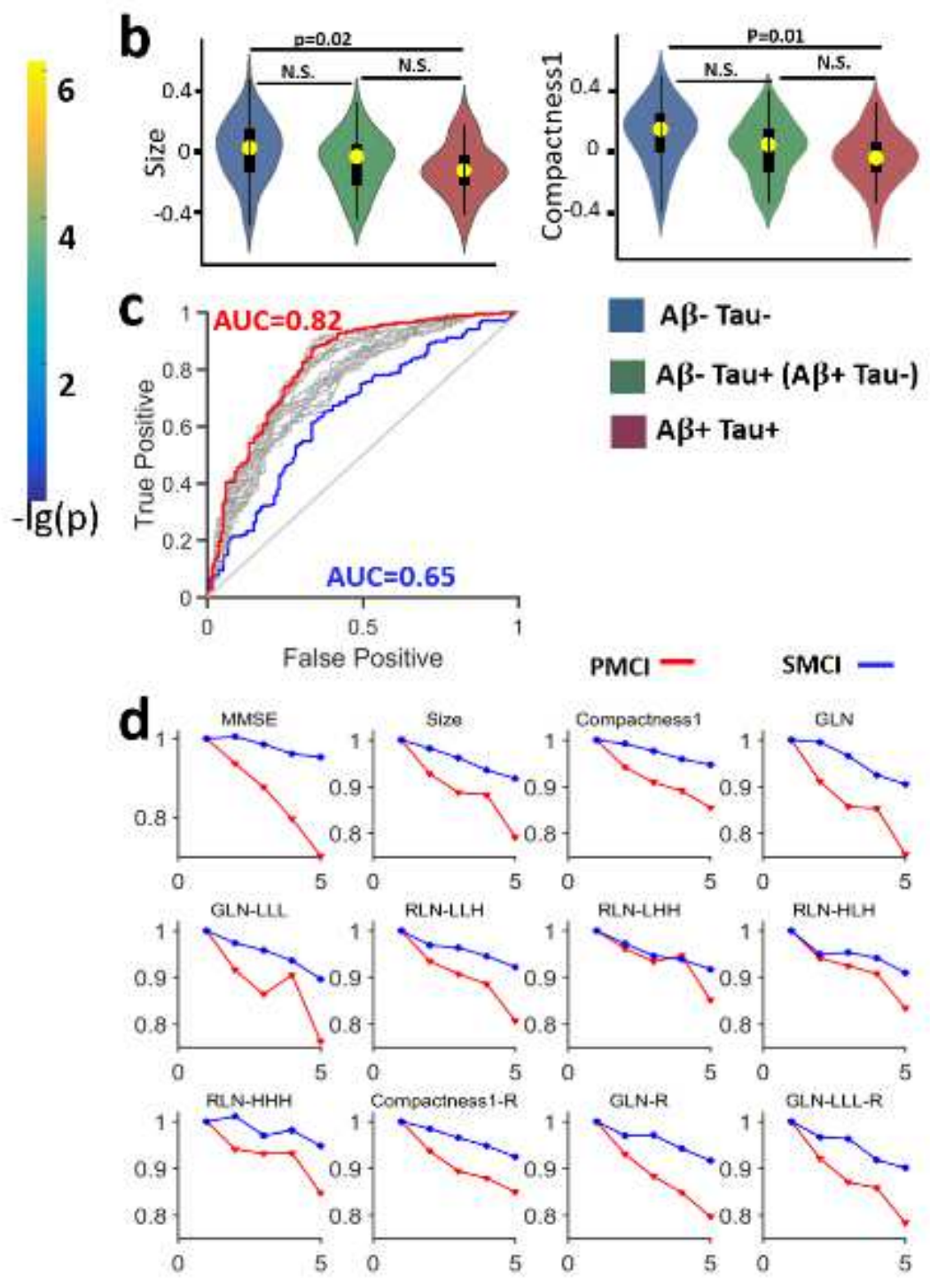

\section{Figure 5}

(a) Negative logarithm of the $p$-value for either a t-test of the group difference or correlation with radiomic features for the following variables: a1, APOE $\varepsilon 4+$ group vs APOE $\varepsilon 4$ - group in the $\mathrm{MCl}$ subjects; a2, correlation with the polygenic risk scores; a3, high $A \beta(A \beta-)$ vs low $A \beta(A \beta+)$ in the $M C l$ subjects; a4, high Tau (Tau+) vs low Tau (Tau-) in the MCl subjects; a5: (A $\beta+\& T a u+)$ vs (A $-\& T a u-)$; a6: correlation between the radiomic features and $A \beta$ in the $\mathrm{MCl}$ subjects, a7: correlation between the radiomic features and Tau in the $\mathrm{MCl}$ subjects; a8: correlation between changes in cognitive ability and changes in features in the $\mathrm{PMCl}$ group; a9: correlation between changes in cognitive ability and changes in features in the $\mathrm{SMCl}$ group. (b) Examples of feature distributions in the subgroups $A \beta+\& T a u+, A \beta+\& T a u-$ or $A \beta-\& T a u+$ and $A \beta-$ \&Tau-. (c) The ROC curve for prediction of whether $\mathrm{MCl}$ conversion to $A D$ increases from $A U C=0.65$ (using age, gender and APOE status) to 0.80 (adding the subset of consistent radiomic features one by one until the AUC does not increase) using logistical regression (see S08 for detailed information). (d) Trend of MMSE changes and the changes in the identified features over 5 years in $\mathrm{PMCl}$ (red) and SMCl (blue). 
Abbreviations: in (a), -L means left hippocampus features, $-\mathrm{R}$ means right hippocampus features; in (d) $\mathrm{PMCl}=$ progressive $\mathrm{MCl}$; $\mathrm{SMCl}=$ stable $\mathrm{MCl}$. 